\title{
Developing Energy-Efficient Topologies and Routing for Wireless Sensor Networks
}

\author{
Hui Tian, Hong Shen, and Teruo Matsuzawa \\ Graduate School of Information Science, \\ Japan Advanced Institute of Science and Technology, \\ hui_t@jaist.ac.jp
}

\begin{abstract}
The performance of wireless sensor networks (WSNs) is greatly influenced by their topology. WSNs with patterned topologies can efficiently save energy and achieve long networking lifetime. In this paper, we discuss different patterned topologies for constructing WSNs which can provide the required coverage area and the connectivity of all sensor nodes. We compare different performance measures among all patterned topologies, and find that WSNs in strip-based topology can provide the greatest coverage area with the same number of sensor nodes as used for WSNs in other patterned topologies. Strip-based topology also consumes least energy in the routing protocol of flooding. We show that triangle-based topology can provide the highest reliability among all patterned topologies. We also propose several routing protocols for WSNs in patterned topologies, which are based on different parameters when selecting next-hop neighbor. Our protocols require only local information and work in a simple and effective way to achieve energy efficiency.
\end{abstract}

Keywords: Wireless sensor networks, topology, routing, energy efficient.

\section{Introduction}

Due to the wide applications of WSNs and inherent limitations of sensor nodes on power and memory, study on energy-efficient topologies and routing becomes increasingly important for WSNs, especially in the scenarios of emergency treatment, disaster rescue and so on. All issues in WSNs are related to one fundamental and important issue, that is how to keep connected coverage in a WSN with as less power consumption in routing as possible.

The connected-coverage problem is to achieve two goals when deploying sensor nodes: coverage and connectivity [4. Coverage is to ensure that the entire physical space of interest is within the sensing range of at least one of the active sensors. Connectivity is to ensure that all the sensor nodes can communicate with the base station by either single-hop or multi-hop path. The connectedcoverage problem in WSNs can easily be solved if the number of sensor nodes and energy-constraint needn't to be concerned. However, it is not possible to construct a connected-coverage WSN without energy and economy concerns in 
practice. Energy-constraint, instead, is extremely stringent in WSNs. Therefore, it is significant to study on constructing a connected-coverage WSN while consuming as least energy as possible so as to maximize the networking lifetime.

Networking lifetime is defined as the time internal from the start to the time that the WSN can provide satisfactory performance on sensing and transmission. It is directly affected by power consumption in the procedure of sensing, communication and data processing. Since all the sensor nodes are battery-powered, it is paramount to develop efficient methods to save energy. The existing work to save energy can be classified into two categories. One is turning off or changing some nodes to sleep mode as proposed in 3410. The other is minimizing sensing range and transmission range while keeping connected coverage as proposed in 58 .

We address the issue of keeping connected coverage with power efficiency from the topology point of view in the paper. It is unlike existing studies in either connectivity and coverage problems, or in energy-efficiency routing protocol. WSNs in patterned topologies are assured to provide longer network lifetime than randomly deployed WSNs if both use the same number of sensor nodes. We call these patterned topologies energy efficient topologies. They have many significant applications. For example, node placement in patterned topologies can efficiently save energy and achieve long networking lifetime in some scenarios where priori node deployment for WSNs are possible. Such scenarios exist in many applications such as danger alarm and vehicle tracking as we have discussed in [1]. Thus study on energy efficiency topologies can guide to construct WSNs with potentially more energy saving and longer lifetime. It is also worth noting that patterned topologies can also instruct to choose duty-on nodes to keep connected coverage in a WSN and put all other sensor nodes in sleeping mode so as to avoid redundant overlapping area, save energy, and thus prolong the networking lifetime 44. Therefore, in this paper, we study different patterned topologies for WSNs and compare their performance on different measures.

We also propose several routing protocols for WSNs with patterned topologies and compare their performance by simulation. This work provides a supplement to 4] which only address how to save energy by choosing duty-on sensor nodes based on patterned topologies. The routing protocols proposed in this paper requires only local information, which is different from DSAP in 9]. Our routing protocols can achieves energy efficiency and perform in a simple and effective way.

The rest of the paper is organized as follows. In section 2 we present the model of connected-coverage WSNs and highlight the important parameters that affect the performance of WSNs. Section 3 compares different topology patterns and their affections on performance of WSNs. Section 4 proposes several routing protocols and compare their performance by simulation. Section 5 gives the concluding remarks. 


\section{Modelling Connected-Coverage WSNs}

A connected-coverage WSN is defined as a wireless sensor network that can guarantee coverage of all the required region and connectivity among all sensor nodes in the WSN. We assume the region of interest to be 2-dimensional. Assume the area of the region to be $A$. There are $N$ sensor nodes and one base station placed in the region. Each sensor node deployed in the region can sense any event within the disk with radius $r_{s}$ centered at the sensor node. Each sensor node can communicate with other sensor nodes whose Euclidean distance between them is no more than $r_{t}$, that is, nodes $s_{1}$ and $s_{2}$ can communicate with each other if their Euclidean distance $E d\left(s_{1}, s_{2}\right) \leq r_{t}$. Otherwise, they cannot. The sensing radius of a sensor node can be either equal or unequal to its communication radius in the WSN.

The sensing ability of each sensor node diminishes as distance increases. In [6], the sensing ability at point $y$ of sensor node $s_{i}$ is assumed to be inversely proportional to $E d\left(s_{i}, y\right)^{k}$ where $k$ is a sensor technology-dependent parameter. This characteristic of sensor nodes introduces an important parameter, we call it sensing strength factor $d_{m m}$, stating how well region $A$ is covered and sensed. If we define $\min _{i} E d\left(s_{i}, y\right)$ as the distance of point $y$ to its closest sensor node, $y \in A$, then all points in $A$ have a distance at most $\max _{y \in A} \min _{i} E d\left(s_{i}, y\right)$. We use $d_{m m}$ to denote this distance:

$$
d_{m m}=\max _{y \in A} \min _{i} E d\left(s_{i}, y\right) .
$$

Thus $d_{m m}$ is the maximum distance from any point to its closest sensor node. Usually a WSN is required to be deployed with a particular sensing strength factor equal to $d_{m m}$ so that distance from any point to its closest sensor node is no more than $d_{m m}$ to ensure coverage and sensing strength. The less $d_{m m}$ is, the better each point is sensed in the WSN. In 2] and [7, similar parameters can be found, but they were proposed for other applications.

The power consumption is another important parameter to measure how much energy different topology patterns can save for WSNs. Since each sensor node usually includes a sensing unit, a processing unit, a transceiver unit and a power unit as modelled in [1], power consumption can be divided into three domains: sensing, communication, and data processing. Of the three domains, we are only concerned with the maximum energy spent by a sensor node in data communication. This involves both power consumed in data transmission, denoted by $P_{t}$, and in data reception, denoted by $P_{r}$. That is, the power consumed by a sensor node is $P_{s}=P_{t}+P_{r}$.

\section{Patterned Topologies for Connected-Coverage WSNs}

As we have discussed in in 11], sensor nodes can be placed in hexagon, square, and triangle-based topologies. In 4 strip-based topology has also been proposed to place nodes to construct a connected-coverage topology for WSNs. We will discuss all these topology patterns in this section and compare the performance 
of WSNs in different patterns. The case that the sensing range of a sensor node equals to its transmission range is discussed in the comparison part.

\subsection{WSNs with Hexagon-Based Topology}

In hexagon-based WSNs, each sensor node has three neighbor nodes located uniquely around the node. Connecting all sensor nodes to their neighbor nodes obtains the minimum unit in the shape of hexagon. Thus the WSN in this topology pattern is called the hexagon-based WSN. The distances of the node to its neighbor nodes are all set to $r_{t}$ so that direct communication is available between the node and its neighbor nodes, and each neighbor provides maximal additional sensing area 11. Figure 1 specifies a WSN with hexagon-based topology. To compare it with other WSNs with different topologies, we place the same number of sensor nodes (25) for all WSNs. We assume node 06 to be the aggregation node which plays the role of aggregating the sensed information in the WSN and reporting to the base station.

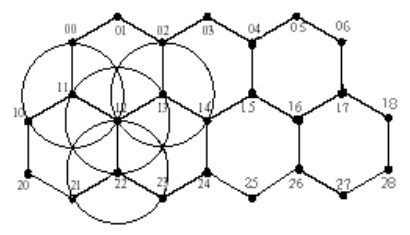

Fig. 1. A WSN with hexagon-based topology

\subsection{WSNs with Square-Based Topology}

In square-based WSNs, each sensor node has four neighbor nodes located uniquely around the node. Connecting all sensor nodes to their neighbor nodes obtains the minimum unit in the shape of square. Thus the WSN in this topology pattern is called the square-based WSN. The distances of the node to its neighbor nodes are set to $r_{t}$. A WSN composed of 25 sensor nodes is given in Figure 2. Node 04 is assumed to be the aggregation node.

\subsection{WSNs with Triangle-Based Topology}

In triangle-based WSNs, each sensor node has six neighbor nodes located uniquely around the node. Connecting all sensor nodes to their neighbor nodes obtains the minimum unit in shape of triangle. Thus the WSN in this topology pattern is called the triangle-based WSN. Same as above, the distances of the node to its neighbor nodes are set to $r_{t}$. Figure 3 is a triangle-based WSN with 25 sensor nodes deployed. Node 04 is assumed to be the aggregation node. 


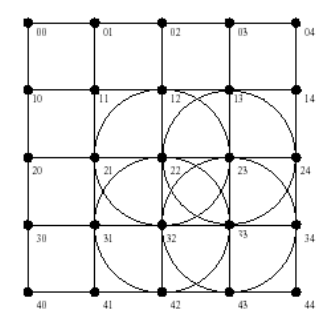

Fig. 2. A WSN with square-based topology

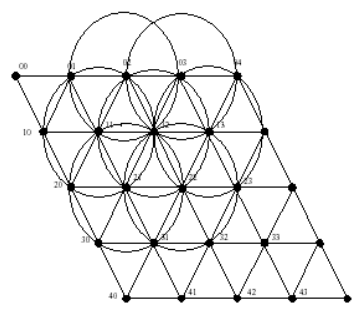

Fig. 3. A WSN with triangle-based topology

In WSNs with triangle-based topology, we find that every point within the area is covered by at least two sensor nodes. We call the reliability provided by such kind of node placement 2-reliability. A 2-reliability WSN can maintain its connected-coverage for any single sensor node failure. When every point is covered by at least $k$ sensor nodes, the sensor network is called $k$-reliability. The WSNs with other patterned topologies are 1-reliability as discussed in 11.

\subsection{WSNs with Strip-Based Topology}

To keep the connectivity of two sensor nodes, their distance should be no more than $r_{t}$. To maximize the coverage area sensed by the same number of sensor nodes, 4 proposes a strip-based topology as in Figure 4.

The strip-based WSN in Figure 4 clearly shows that sensor nodes 40, 41 and 42 connect 4 self-connected strips $00-05,10-14,20-25$ and $30-34$. By this way of node placement, these sensor nodes construct a connected WSN with strip-based topology. The total number of sensor nodes is 25 as before. We assume node 05 to be the aggregation node.

\subsection{Performance Comparison}

Given the same number of sensor nodes, we compare the above four types of patterned topologies on coverage area, sensing strength factor, reliability and energy consumption. We assume $r_{t}=r_{s}=r$ in all WSNs. For coverage area 


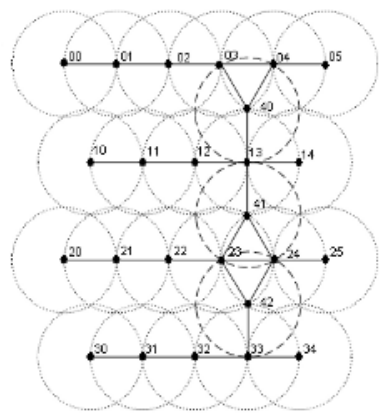

Fig. 4. A WSN with strip-based topology

\begin{tabular}{|c|c|c|c|c|}
\hline Topology pattern & $\begin{array}{c}\text { Coverage area by } \\
25 \text { sensor nodes } \mathrm{A}\end{array}$ & $\begin{array}{c}\text { Sensing strength } \\
\text { factor } \mathrm{d}_{\mathrm{mm}}\end{array}$ & Reliability & $\begin{array}{c}\text { Energy consumption } \\
\text { by flooding } \mathrm{PS}\end{array}$ \\
\hline $\begin{array}{c}\text { Hexagon-based } \\
\text { topology }\end{array}$ & $32.48 \mathrm{r}^{2}$ & $\mathrm{r}$ & 1 & $24 \mathrm{P}_{\mathrm{t}}+60 \mathrm{P}_{\mathrm{r}}$ \\
\hline $\begin{array}{c}\text { Square-based } \\
\text { topology }\end{array}$ & $25 \mathrm{r}^{2}$ & $0.71 \mathrm{r}$ & 1 & $24 \mathrm{P}_{\mathrm{t}}+78 \mathrm{P}_{\mathrm{r}}$ \\
\hline $\begin{array}{c}\text { Triangle-based } \\
\text { topology }\end{array}$ & $21.65 \mathrm{r}^{2}$ & $0.58 \mathrm{r}$ & 2 & $24 \mathrm{P}_{\mathrm{t}}+110 \mathrm{P}_{\mathrm{r}}$ \\
\hline $\begin{array}{c}\text { Strip-based } \\
\text { topology }\end{array}$ & $46.65 \mathrm{r}^{2}$ & $\mathrm{r}$ & 1 & $24 \mathrm{P}_{\mathrm{t}}+53 \mathrm{P}_{\mathrm{r}}$ \\
\hline
\end{tabular}

Fig. 5. Performance comparison among WSNs with different patterned topologies

comparison, we do not consider the marginal places covered by edge sensor nodes because the marginal area exists only in a few places and occupies a negligible portion of the whole coverage area of the WSN which often includes a huge number of sensor nodes. For energy consumption comparison, we fix the destination to be the aggregation node as designated above. The source is fixed to be a node with distance $4 r$ from the destination. In this case, the less the energy is consumed, the better the node placement pattern is. The table in Figure 5 gives the results of these performances comparison.

From the table in Figure 5, we can see that strip-based topology provides maximal connected-coverage with the same number of sensor nodes and consumes least energy by the routing protocol of flooding. WSNs in triangle-based topology provide the best reliability and the best sensing strength while trading off total coverage area and energy consumption. These conclusions hold when comparison is performed in general cases of large-scale WSNs.

\section{Routing Protocols in Patterned WSNs}

We propose several routing protocols in this section. Different from Directional Source-Aware Protocol (DSAP) 9] where each node must have the knowledge 


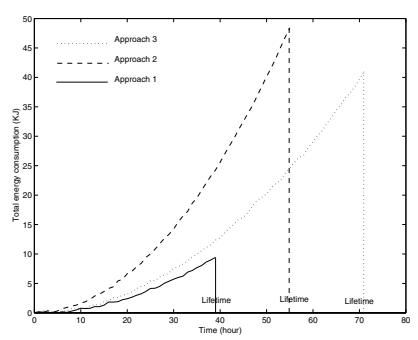

Fig. 6. Energy consumption and lifetime comparison among three approaches

of global information of topology, our routing protocols only require local information.

We define a routing selection function $f(h, s)$ for a sensor node to choose neighbor nodes when routing the message back to the aggregation node. The function is determined by the hop count value $h$ of neighbor nodes and stream unit $s$ which has been sent by neighbor nodes. Here we assume the stream sent by a sensor node can be measured by stream unit, thus $s$ means how many units have been sent by the sensor node. We denote the battery life of sensor node $i$ by $b_{i}$.

We propose three approaches to route back the message for different aims. All of them are based on the routing selection function $f(h, s)=\alpha h+\beta s$.

1. Maximize the total energy saving for WSNs, i.e., $B=\sum_{i} b_{i}$ : This can be obtained by minimizing first the hop count value $h$ when choosing next-hop neighbor and then minimize $s$. In this case, $\alpha=1$, and $\beta=\sigma$, where $\sigma$ is a small number which approximates to 0 .

2. Maximize the minimal energy maintained by all sensor nodes, i.e., $\min _{i} b_{i}$ : This can be obtained by minimizing first the stream units $s$ of next-hop neighbor and then $h$. In this case, $\alpha=\sigma$, and $\beta=1$.

3. Maximize both the total energy and minimal energy of all sensor nodes: This can be obtained by minimizing both $h$ and $s$. In this case, $\alpha=\beta=1$.

We name the protocols as routing selection function-based protocols. It works as follows:

1. Distance identification: The aggregation node floods the discovery message in the WSN with a determined $T T L$ value. Each sensor node records its distance from the aggregation node by hop count. If a sensor node receives several broadcast messages, it records the least value of hop count.

2. Data collection: When a sensor node senses any abnormal event and needs to report the event, it chooses a neighbor with minimized $f(h, s)$ to route back the message.

To compare the performance of our protocols, we simulate the square-based WSN with the routing protocol for simplicity. We use OPNET as the simulation environment. We assume the networking lifetime is from the start to the time 
that any node exhausts its power in the WSN since one node failure results in an unconnected coverage for WSNs with square-based topology.

From Figure 6, we can see that Approach 1 provides least network lifetime. Approach 2 gets a longer lifetime than approach 1 and, however, trades off much more energy consumption by choosing a longer path to the aggregation node in the WSN. Approach 3 can provide the longest lifetime, which is almost as twice as that provided by Approach 1 because it tries to find a shorter path and a next-hop neighbor with more energy in every step.

\section{Conclusion}

We discussed different patterned topologies for WSNs in this paper. It is found that strip-based topology provides the maximal connected coverage and consumes the least energy by flooding protocol, whereas triangle-based topology reaches the best coverage performance with a higher reliability and greater sensing strength. In WSNs with patterned topology, we proposed several routing protocols which achieve different goals. The simulation showed that the networking lifetime is maximized by selecting routing based on both hop count and stream unit of next-hop neighbor. Thus, patterned WSNs equipped with their protocols provide great promises and guarantees their potential applications to meet different needs. Our routing protocols may also be extended to WSNs without regular topologies, which will be explored in our future study.

\section{Acknowledgement}

This research is conducted as a program for the "21st Century COE Program" by Ministry of Education, Culture, Sports, Science and Technology.

\section{References}

1. I.F. Akyildiz and W. Su, Y. Sankarasubramaniam and E. Cayirci, Wireless Sensor Networks: A Survey, Computer Networks, Vol. 38(4), pp.393-422, 2002.

2. Edoardo S. Biagioni and Galen Sasaki, Wireless Sensor Placement For Reliable and Efficient Data Collection, Proc. of the 36th Hawaii International Conference on System Sciences (HICSS), 2003.

3. Alberto Cerpa and Deborah Estrin, ASCENT: Adaptive Self-Configuring sEnsor Networks Topologies, Proc. of INFOCOM, 2002.

4. Koushik Kar and Suman Banerjee, Node Placement for Connected Coverage in Sensor Networks, Proc. of WiOpt, 2003.

5. Martin Kubisch and Holger Karl and Adam Wolisz and Lizhi Charlie Zhong andJan Rabaey, Distributed Algorithms for Transmission Power Control in Wireless Sensor Networks, Proc. of IEEE Wireless Communicationsand Networking Conference (WCNC), 2003.

6. Xiang-Yang Li and Peng-Jun Wan and Ophir Frieder, Coverage in Wireless Ad-hoc Sensor Networks, Proc. of IEEE ICC, 2002. 
7. Seapahn Meguerdichian and Farinaz Koushanfar and Miodrag Potkonjak and Mani B. Srivastava, Coverage Problems in Wireless Ad-hoc Sensor Networks, Proc. of IEEE INFOCOM, 2001.

8. Jianping Pan and Y. Thomas Hou and Lin Cai and Yi Shi and Sherman X. Shen, Topology Control for Wireless Sensor Networks, Proc. of MobiCom, 2003.

9. Ayad Salhieh and Jennifer Weinmann and Manish Kochhal and Loren Schwiebert, Power Efficient Topologies for Wireless Sensor Networks, Proc. of Int'l Conf. on Parrallel Processing, 2001.

10. Di Tian and Nicolas D. Georgannas, A Coverage-Preserving Node Scheduling Scheme for Large Wireless Sensor Networks, Proc. of ACM Int'l Workshop on Wireless Sensor Networks and Applications(WSNA), 2002.

11. Hui Tian and Hong Shen, An optimal coverage scheme for wireless sensor network, Proc. of 2005 IEEE International Conference on Networks (ICN'05), Reunion Island, France, April 2005, pp. 722-730. 